\title{
Modelling of weather radar echoes from anomalous propagation using a hybrid parabolic equation method and NWP model data
}

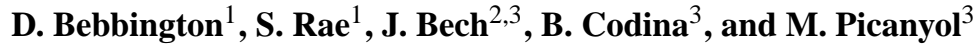 \\ ${ }^{1}$ Dept. Electronic Systems Engineering, University of Essex, UK \\ ${ }^{2}$ Servei Meteorològic de Catalunya, Barcelona, Spain \\ ${ }^{3}$ Dept. d'Astronomia i Meteorologia, Universitat de Barcelona, Spain
}

Received: 10 January 2006 - Revised: 1 March 2007 - Accepted: 20 March 2007 - Published: 8 June 2007

\begin{abstract}
Contamination of weather radar echoes by anomalous propagation (anaprop) mechanisms remains a serious issue in quality control of radar precipitation estimates. Although significant progress has been made identifying clutter due to anaprop there is no unique method that solves the question of data reliability without removing genuine data. The work described here relates to the development of a software application that uses a numerical weather prediction (NWP) model to obtain the temperature, humidity and pressure fields to calculate the three dimensional structure of the atmospheric refractive index structure, from which a physically based prediction of the incidence of clutter can be made. This technique can be used in conjunction with existing methods for clutter removal by modifying parameters of detectors or filters according to the physical evidence for anomalous propagation conditions. The parabolic equation method (PEM) is a well established technique for solving the equations for beam propagation in a non-uniformly stratified atmosphere, but although intrinsically very efficient, is not sufficiently fast to be practicable for near real-time modelling of clutter over the entire area observed by a typical weather radar. We demonstrate a fast hybrid PEM technique that is capable of providing acceptable results in conjunction with a high-resolution terrain elevation model, using a standard desktop personal computer. We discuss the performance of the method and approaches for the improvement of the model profiles in the lowest levels of the troposphere.
\end{abstract}

\section{Introduction}

Weather radar echoes affected by anomalous propagation (anaprop) mechanisms remains a serious issue in quality control of radar products and, particularly, in subsequent precip-

Correspondence to: D. Bebbington

(david@essex.ac.uk) itation estimates (Joss and Waldvogel, 1990; Collier, 1996; Collier and Hardaker, 2003). Although significant progress has been made in terms of identifying clutter due to anaprop, including Doppler (Doviak and Zrnic, 1993), texture (Krajewski and Vignal, 2001; Sánchez-Diezma et al., 2001; Haddad et al., 2004), height profiling (Alberoni et al., 2001), or multi-sensor methods (Pamment and Conway, 1998; Michelson and Sunhede, 2004) there is no unique method that solves the question of data reliability without excessively removing genuine data. Better results can be obtained using polarisation diversity measurements (Illingworth, 2003) though most radar systems are still limited to single polarisation observations.

Built on previous studies (Bebbington, 1998), the work described here relates to the development of a methodology to simulate radar echoes and its implementation as a software application that uses numerical weather prediction (NWP) model data as input. NWP data provide the temperature, humidity and pressure fields to model the three dimensional structure of the atmospheric refractive index structure, from which a physically based prediction of the incidence of clutter can be made. This technique can be used in conjunction with existing methods for clutter removal based on post-processing of observations according to the physical evidence for anomalous propagation conditions and diagnostic or prognostic anaprop products also based on NWP output (Bech et al., 2006).

The backbone of the methodology described in this paper lies in the use of the parabolic equation method. The parabolic equation method is a well established technique for solving the equations for beam propagation in a nonuniformly stratified atmosphere, but although intrinsically very efficient, is not sufficiently fast to be practicable for near real-time modelling of clutter over the entire area observed by a typical weather radar. We demonstrate a fast hybrid technique that is capable of providing acceptable results in conjunction with a high resolution terrain elevation

Published by Copernicus GmbH on behalf of the European Geosciences Union. 
model, using a typical desktop personal computer. We discuss the performance of the method and approaches for the improvement of the model profiles in the lowest levels of the troposphere.

\section{Modelling radar echoes with the Parabolic Equation Method}

The key to the efficient modelling of the electromagnetic is an adaptation of the well established parabolic equation method (PEM). This can be made ultra-efficient by recognising the role of Huygens' Principle in the operation of the PEM, so that, for a radar beam of relatively narrow beam width (i.e. around 1 degree), an adequate spatial discretization, capable of resolving vertical variations in refractive index involves and angular discretization of the wavefront that is in the range $10^{2}-10^{3}$ elements - much smaller than is typical with PEM techniques. For this particular application, coherent integration of incident and reflected fields is not required, since the targets of interest are distributed and incoherent. The following subsections present the most relevant aspects of the methodology developed. Further mathematical details can be found in Appendix A and a description of the implementation of the software is covered in Appendix B.

\subsection{Effects of the air 3-D refractivity field}

The principal phenomenon that is being modelled is the effect of the refractivity structure in the atmosphere, which is determined by the thermodynamic parameters, temperature, humidity and pressure. For the most part, these quantities are horizontally stratified, although horizontal gradients while much smaller are significant, since anomalous propagation arises when all or part of a radar beam is close to grazing incidence with a stratification that refracts the local wavefront towards the ground.

The aim of the methodology presented is to incorporate the spatial modelling of the thermodynamic fields in an NWP model into the propagation model. The Mesoscale Atmospheric Simulation System (MASS model) was used for this purpose (see Koch et al., 1985; Codina et al., 1997a, b for details). In the data used gridpoint spacings are of the order of $8 \mathrm{~km}$ in the horizontal, and 32 unequally spaced levels from the surface to the top level. For the purposes of the propagation modelling, it is the lowest levels that are generally significant for anomalous propagation. Operational MASS model runs have provided datasets from which the refractivity fields could be produced, based on test cases identified by SMC from radar reflectivity images and radiosonde observations, including cases of anaprop without precipitation, precipitation only, and mixed cases.

The proposed methodology has been constructed on the basis of the use of cubic spline approximations of the local vertical refractivity. This allows very rapid integration of the ray-paths used to construct the reference mean wave surfaces. It is important to stress that the ray paths only need to be modelled approximately; small errors lead to (re)emergence of apparent refractive index structure which then causes a corrective beam bending. The overall modelling of the refractivity structure is therefore a two-stage process: firstly, the sigma-coordinate surfaces of the original NWP model are used to construct a grid of refractivity-height pairs for all the sigma-levels. A two-dimensional interpolation procedure - a modified Akima method - is used to resample these at suitable intervals on the radials of the radar coordinate system. The Akima method (Akima, 1991) is a form of interpolation - less efficient than B-splines - that has the goal of maximizing smoothness, and in particular avoiding spurious overshoots. This approach was adopted because such overshoots would misrepresent beam clearances and exaggerate roughness. The lower efficiency is of little concern, since this task is performed once only for a given domain. After the interpolation, vertical spline approximations are constructed. This preserves the vertical detail of the original model data.

\subsection{Effects of terrain}

The terrain of the area studied (Catalonia, located in the NE of Spain) was described with a DEM with a resolution on the order of $30 \mathrm{~m}$ in geographical coordinates and vertical resolution of $1 \mathrm{~m}$. Such a high resolution is important in order to be able to interpolate the terrain to a polar radial grid based on the radar coordinate frame without introducing spurious features, and in order to be able to make realistic models of terrain slope, to which the coherent forward scatter and incoherent backscatter intensity calculations are very sensitive. Careful consideration had to be given to the representation of terrain, as the quantities of data involved at such resolution are significant in relation to available memory in current typical personal computers (100 s of Megabyte). A representation that combined sufficient compactness with the required flexibility was found to be a B-spline. Some experimentation was undertaken using the Catalonia dataset, to gauge appropriate ways to fit the terrain. A method was adopted based on the work of Dierckx (1995) to provide B-spline fits constrained by a smoothing parameter. This permits a reasonably tight fit to the terrain profile while suppressing spurious oscillation that can occur through exact fitting.

\subsection{Modelling of terrain coherent reflection}

As it is well known, incidence at near grazing angles results, even for random rough surfaces, in near coherent quasispecular reflection when the Rayleigh criterion is achieved. This means, for incidence at close to one degree, roughness scales of tens of wavelengths - at C-band, therefore of up to about $2 \mathrm{~m}$ in standard deviation of the height can be regarded as smooth. It is therefore reasonable to model the 
forward propagation on incidence with either sea or terrain by means of a smooth curve representation of the local topography. Rigorous asymptotic methods for scattering from a rough interface between two dielectric half spaces show that the far-field contributions may be constructed by means of geometric phase factors associated with reflection from the local tangent planes combined with local Fresnel reflection coefficients. In addition, there may be resonant terms, but we do not evaluate these as they are not related to propagating modes.

A major challenge for the implementation of the model was to preserve the efficiency of the hybrid ray/PEM technique while evaluating the reflection from a randomly undulating terrain. This was neatly solved by consideration of the orthotomic image of the source with respect to the terrain profile. The orthotomic is constructed as the image of a point (in effect the centre of curvature of the mean incident wavefront) in the envelope of tangent planes to the surface. Thus, the required phase factor in the integral can be realised by constructing virtual sources on the orthotomic curve.

In general, one would expect the orthotomic curve of a smooth, random surface with respect to a general point to be itself a highly random curve. However, the typical geometry of near grazing incidence (which is the most significant case for anomalous propagation) ensures that the points of the orthotomic lie close to a surface of constant curvature (Fig. 1).

The key step that allows the terrain contributions to be evaluated by essentially the same technique as that for the direct radar beam is to interpolate data to the mean orthotomic curve, compensating for the spatial adjustment of the source positions by a phase factor; this is permissible because of the paraxial nature of the wavefront angles. Hence, the propagation of the reflected waves is evaluated by the application of Huygens' Principle in the same way as before. It takes one application of the split-step method to propagate the field forward onto a convex surface close to the point of incidence of the wave with the terrain surface. The total extra computation per step due to terrain interaction is therefore comparable to that of the free-space beam.

In the case of terrain of relatively high relief, only a small portion of the terrain is ever involved in interaction. In the software application, the total field is described by a data structure organised as a tree of "wave surfaces". That is, each set of reflections is handled independently and related to a local propagating wavefront.

For the purposes of the application, it is not generally necessary to evaluate the total coherent field; however, for validation purposes it will be possible to superpose linearly the coherent contributions that are local to any point of interest. In practice, the "tree" does not subdivide geometrically, as downward-propagating wavefronts ultimately are stopped by the terrain, and upward propagating wavefronts only subdivide if they are subsequently bent back towards the ground.
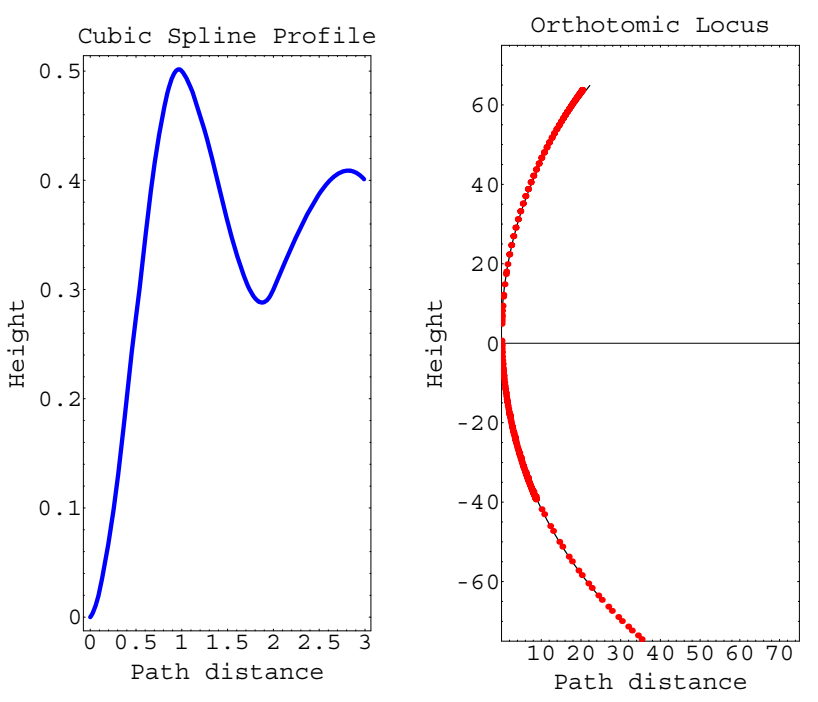

Fig. 1. The left hand graph shows a model curve formed from cubic splines representing the large scale form of hilly terrain; units are arbitrary, but the slopes are actual. On the right is a plot of the collection of points on the orthotomic curve imaged for a point (representing the radar location at 100 units from the terrain section). For reference, a circular arc is plotted close to the points. This illustrates that although the orthotomic images of nearby points are not necessarily close, all the image points lie close to a surface of well defined curvature.

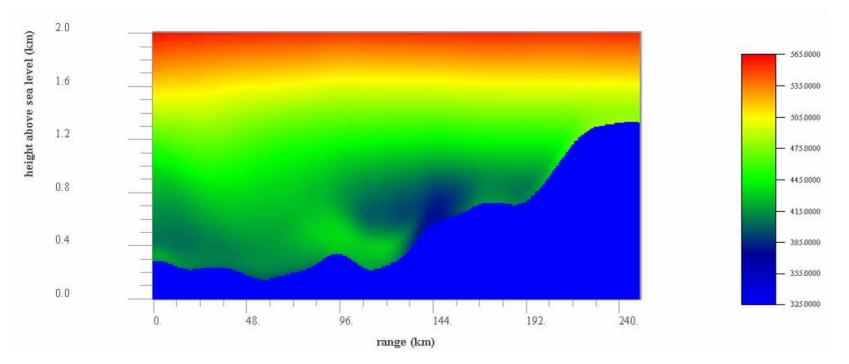

Fig. 2. The figure shows the modified refractive index field M (plotted in N-units) calculated from NWP model output thermodynamic fields for one of the case-study examples, at an azimuth of $250 \mathrm{de}-$ grees. Areas of negative vertical gradient (highlighted) can clearly be seen, which lead to downward curvature of the radar beam.

\section{A case study}

A case study has been selected to illustrate the results of the methodology described in the previous section. The anomalous propagation event simulated took place in the NE of Spain on the 19 July 2003. In mid and late summer intense super-refractive events are quite common in this region (Bech et al., 2000, 2002).

The refractivity field was modelled for the entire volume of the radar scan, and a section at 250 degrees azimuth is shown in Figs. 2, 3, and 5. Figure 2 depicts the modelled 


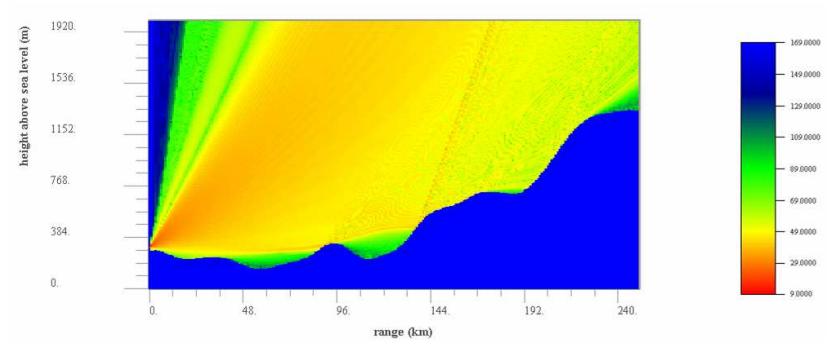

Fig. 3. Modelled one way path transmission loss (in $\mathrm{dB}$ ) from the radar, using the modelled modified refractive index fields of Fig. 2. Curvature of the beam can clearly be seen in the vicinity of the area of negative gradient in M. Local enhancement of the beam intensity can also be discerned.

modified refractive index field, which depends on pressure, temperature and water vapour pressure, plus a linear term of $157 \mathrm{~N}$-units per $\mathrm{km}$ in height, which corrects for the Earth's curvature. In normal conditions the latter term is strong enough to dominate the natural tendency of actual refractive index to fall with height because of reduction in density of both dry air and the moist component. In anomalous propagation conditions, the latter effects due to temperature gradient can be so severe that the $\mathrm{M}$ gradient becomes negative, allowing anomalous propagation to occur. In Fig. 2, a region to the right of centre is particularly strong, and there is also a less pronounced area in the first $30 \mathrm{~km}$ from the radar. Figure 3 shows the modelled one-way pass loss factor from the radar (shown in $\mathrm{dB}$ ). One-way path loss expresses the ratio of transmitter power to that coupled to a remote isotropic antenna. The intensity of the apparent reflectivity from backscatter from terrain depends on the square of the path-loss factor and the intrinsic reflectivity of the ground at the angle of incidence of the lower edge of the beam. Although the latter factor is hard to model accurately without detailed information on the surface roughness height, the uncertainties are somewhat less than the magnitude of variation of the path loss factor. In Fig. 3 it is possible to discern strong filaments in the beam pattern at low levels, which have a large impact on the modelled value of reflectivity, whose variation along the path is shown in Fig. 4a. The increase in ground clutter compared to observations under normal propagation conditions can be appreciated comparing Fig. 4a and Fig. $4 \mathrm{~b}$. The vertical refractivity gradient among surface level and $1 \mathrm{~km}$ above ground observed by a nearby radiosonde station (located $20 \mathrm{~km}$ from the radar) in the two events were $-112 \mathrm{~km}^{-1}$ and $-42 \mathrm{~km}^{-1}$, respectively.

Figure 5 shows a plot of the actual recorded radar reflectivity factor pattern for the example of the case study. It is clear that the regions of enhanced reflectivity along the 250 degree azimuth are in substantial agreement with the model. In particular, the ground clutter echoes between 100 and 150 are remarkably similar, both in position and intensity.

A more complete view is given in Fig. 6 which shows full

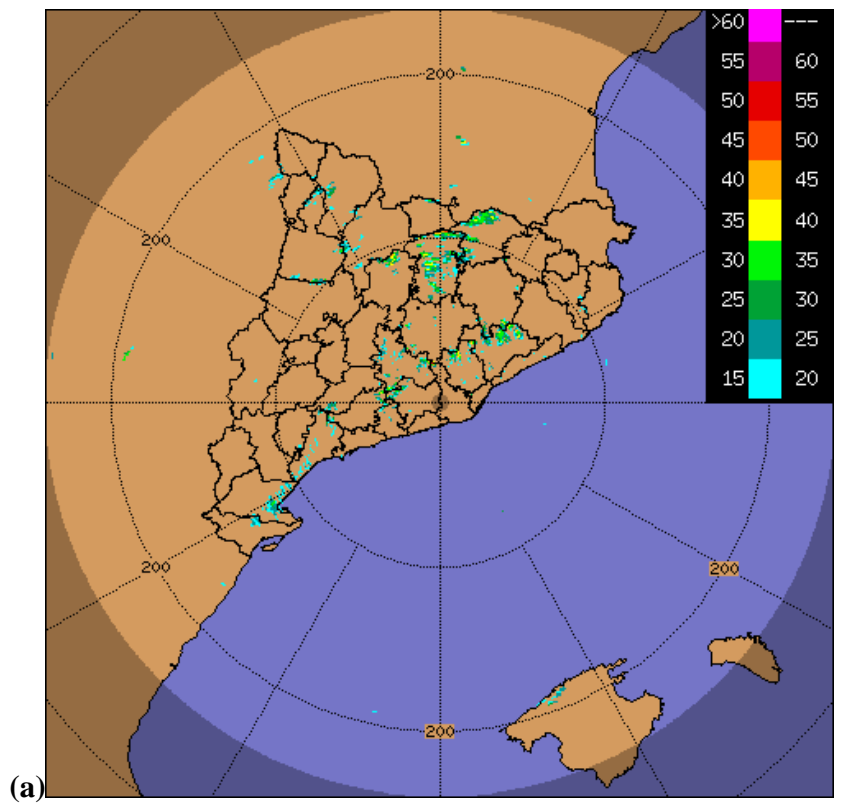

(a)

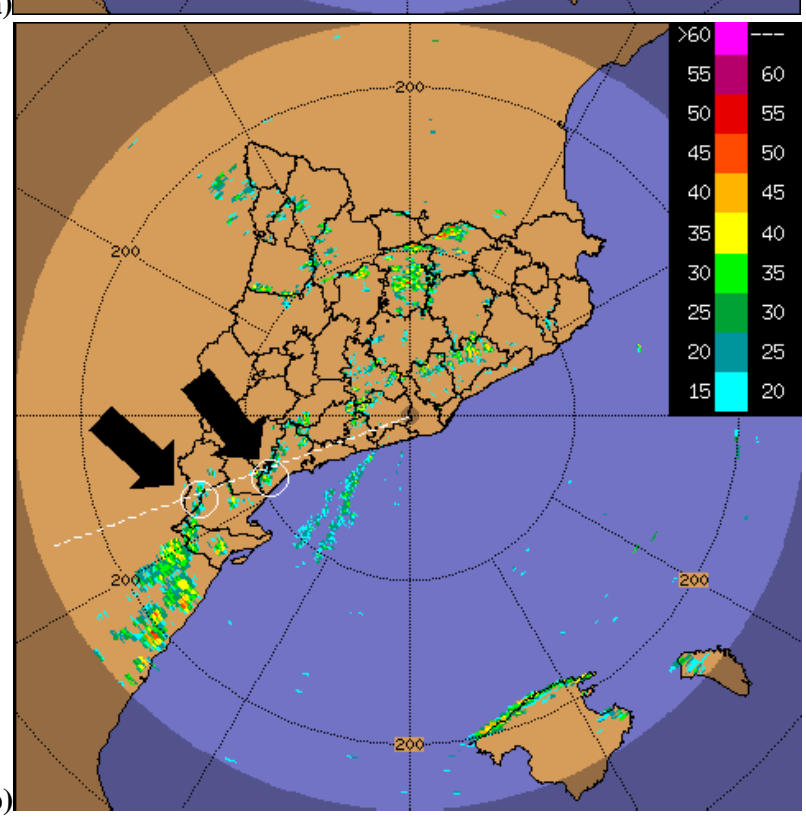

Fig. 4. (a) Actual observed radar reflectivity factor (dBZ) for the case study modelled in Figs. 2 and 3. Agreement with the areas for which enhanced reflectivity is predicted (around $95 \mathrm{~km}$ and $136 \mathrm{~km}$ as indicated by arrows) is good. Predicted high reflectivity at further ranges from $190 \mathrm{~km}$ is not seen at this azimuth, although present in nearby azimuths. As a reference, (b) shows an equivalent PPI observation recorded under normal propagation conditions (21 July 2003 00:30 UTC). Note the lower intensity of ground and sea clutter.

PPI images of the predicted and observed reflectivity patterns and also a reference of clutter pattern for normal propagation conditions. The overall simulated and observed patterns are similar in intensity and spatial distribution, particularly in the first $60 \mathrm{~km}$ from the radar. From there, the pattern to the 


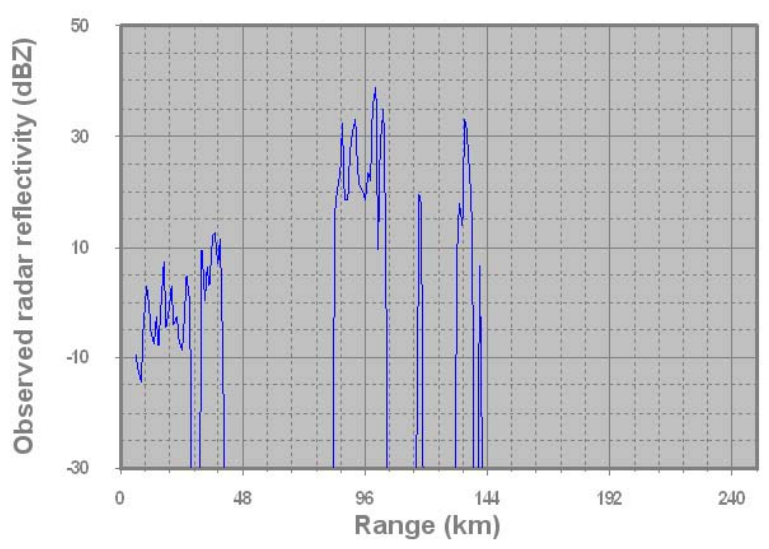

(a)

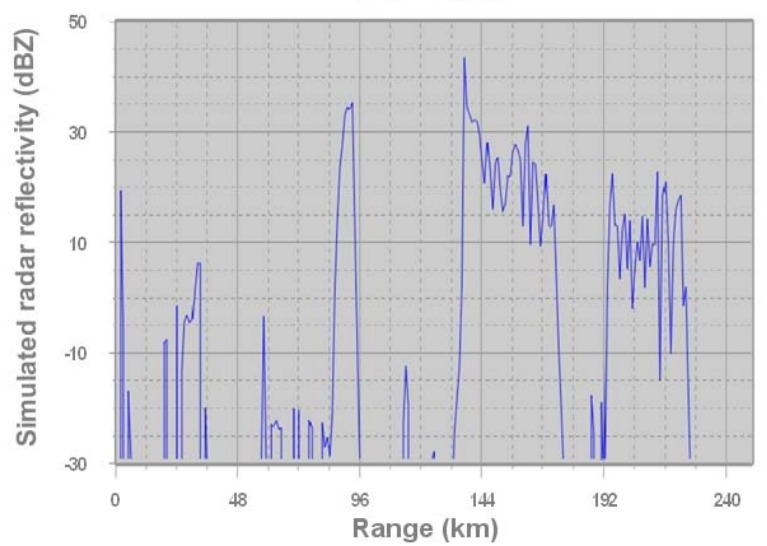

(b)

Fig. 5. Observed (a) and predicted (b) values of radar reflectivity along the path shown in Figs. 2, 3 and 4. Observed reflectivity depends on the path loss factor (each way) as well as the backscatter from the ground. The latter factor is uncertain, because the slope dependence of reflectivity is strongly dependent on surface roughness.

north agrees generally the major sheltered areas. The hills located to the W/SW are also correctly simulated, including the first isolated mountain massif located between 60 and $80 \mathrm{~km}$ to the $\mathrm{W}$ from the radar. The sea clutter area observed to the SW/S is qualitatively indicated in the prediction but located further south. The forecast also overpredicts sea clutter echoes to the east. In fact, though not present in this case in the observation, sea clutter is commonly observed near the coast in that direction in anaprop events (Bech, 2003). It should be noted that the high resolution digital elevation model covered the area of Catalonia (indicated by the counties in Fig. 4) but not the surrounding areas nor the island of Majorca and Minorca, which present ground clutter in $6 \mathrm{~b}$.

These results show the potential of the technique that should be further explored. In general, the most significant expected limitations of this approach derive from the limited vertical resolution of the refractivity field. The basic success of the method suggests that improvements in modelling the

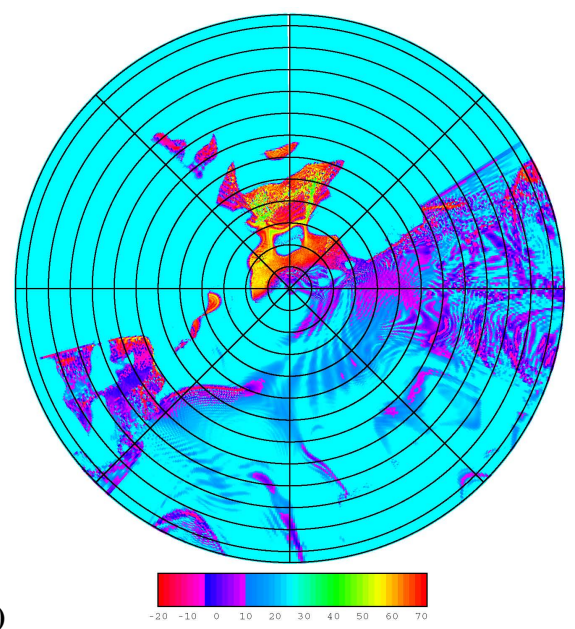

(a)

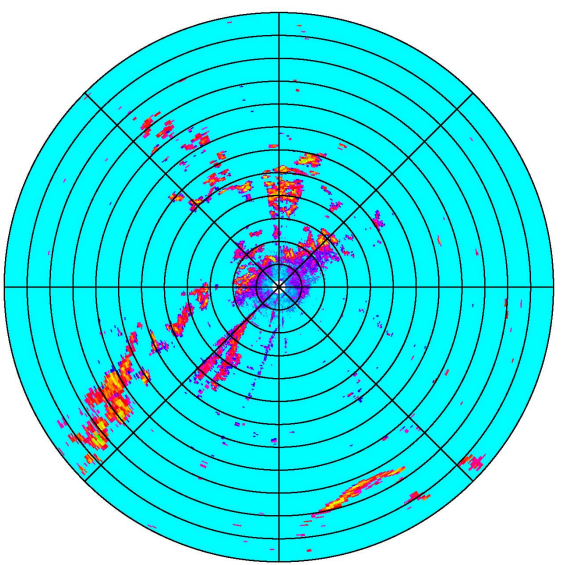

(b)

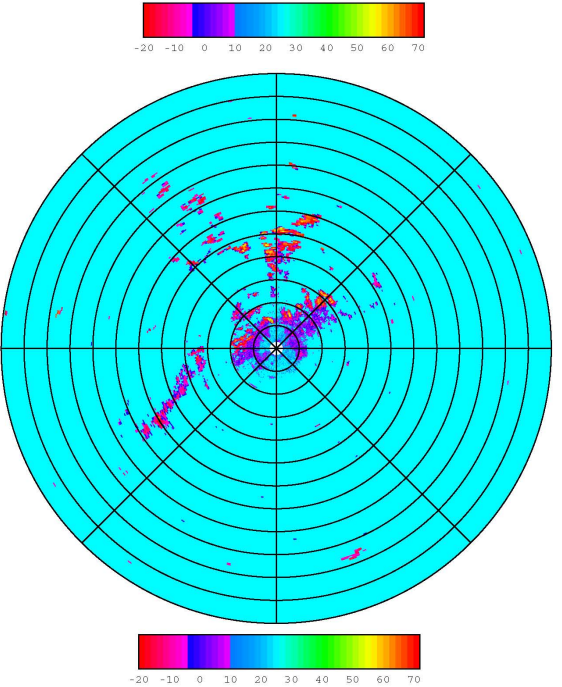

(c)

Fig. 6. (a) Simulated full PPI radar reflectivity factor corresponding to the case study and actual radar observation (19 July 2003 03:36 UTC) shown in Fig. 4a but in the same display format. A normal propagation example showing the usual clutter pattern (Fig. 4b) is also given as a reference. The maximum range represented in (a) is $250 \mathrm{~km}$ and in (b) and (c) is $240 \mathrm{~km}$. 
vertical profile in the first hundred metres would be beneficial.

\section{Summary and conclusions}

The successful implementation of this work should mark an improvement in the identification of clutter due to anomalous propagation in weather radar data, which would in turn lead to useful enhancements in the quality indexes of such data. The modelling system proposed is more realistic than the geometric optics approach of the radar beam propagation assumed typically in radar beam blockage corrections that take into account propagation conditions (Bech et al., 2003; Fornasiero et al., 2005).

This improvement shows potential to contribute to enhance the quality controls in assimilation of rainfall products in hydrological forecasting systems and numerical weather prediction (NWP) models (Alberoni et al., 2003). The need to do so is expected to become increasing acute over the next decade, as the computational capabilities available will permit grid-scales to decrease by almost an order of magnitude in a few years. It is clear that in many forums within the weather forecasting community improvements in the quantitative measurement and forecasting of precipitation will be given increasing priority, since it is believed to be likely that situations with severe impact due to extreme weather events may become more frequent, and the most effective way to mitigate the effects of such events is to marshal resources through decisions based on quality controlled data and understanding of its reliability.

The case study presented illustrates the potential of the technique proposed. Further research is needed to perform a more detailed sensitivity analysis of the various parameters of the propagation model in order to better understand the limitations of this methodology.

Moreover the system described in this paper may also be used to visualise the simulated echoes over terrain model data and then compare them to real radar observations. As described by Curtis et al. (2000) this may provide a deeper understanding of the different interactions between both real and spurious precipitation echoes with terrain features and thus lead to further improvement in the radar observations and precipitation estimates in complex topography environments.

\section{Appendix A}

This appendix contains some mathematical details - not mentioned explicitly in the text - regarding the methodology described in this paper. A full detailed formulation is out of the scope of this paper but interested readers may hopefully find a useful description in this appendix dealing with the most remarkable aspects.
The methodology that has been pursued is to implement the PEM via a hybrid technique that implicitly exploits Huygens' Principle by integrating the phase paths between an initial mean phase front and a target mean phase front.

The PEM 'split-step' methodology combines the effects of stationary phase integration with the accumulation of wavefront distortions due to local variations in refractive index variation. For each step one starts by tracing a small number of ray-trajectories across the wavefront. For a triad of trajectories a ray's congruence may be interpolated so that the second mean wavefront may be constructed. Then, ideally, the phase paths between corresponding points are stationary. In the standard PEM technique, phase integration is estimated with respect to straight-line paths and path-averaged refractive index. In the present case, the phase paths arise from curvature in a non-uniform refractive index gradient. The actual phase variation can be determined implicitly for short arcs by knowledge of the excess geometric paths without explicit integration of the refractivity, since the two contributions are in the ratio $2: 1$.

In this appendix, we show that, to a good approximation, assuming paraxial propagation, the unknown phase integral between any two points can be determined by a knowledge of the refractive index on the start and end surfaces, and an integral of the squared slope of the ray, without any further assumption on the detail of the refractive index structure in between.

In the paraxial approximation we compare the actual ray paths between two points, whose separation along the nominal $\mathrm{x}$ axis is $\mathrm{L}$ with the straight line path. Assuming a ray is propagated between two vertical planes indexed 1,2 and separated by $\mathrm{L}$, we define three integrals between the endpoints of the ray,

$$
\begin{aligned}
& l_{12}=\int_{0}^{L} d r \approx \int_{0}^{L}\left(1+\frac{\vartheta}{2}^{2}\right) d x \\
& r_{12}=\int_{0}^{L} d r \approx \int_{0}^{L}\left(1+\frac{\vartheta}{2}^{2}\right) d x \\
& s_{12}=\int_{0}^{L} n(r) d r \approx \int_{0}^{L}\left(1+\frac{\vartheta}{2}^{2}\right) d x
\end{aligned}
$$

where $l_{12}$ is the straight line path, $r_{12}$ is the integrated length (in space) of the ray, and $s_{12}$ is the integrated optical path dependent on the refractive index $n(r)$ which is path dependent, $\vartheta_{0}$ is the slope of the straight path, and $\vartheta$ is the slope of the ray at each point of the path. According to ray-optics, we find from Snell's law that

$n \cos \vartheta=$ const.

for any ray. In the paraxial approximation, and assuming $n \approx 1$ can express this appropriately as,

$n(\vartheta)=n\left(\vartheta_{*}\right)+\frac{\vartheta^{2}}{2}-\frac{\vartheta_{*}^{2}}{2}$

for any suitable reference $\vartheta_{*}$. 
Table B1. Different software modules for terrain and microphysics/refraction computations.

\begin{tabular}{lll}
\hline & Module & Description \\
\hline \multirow{2}{*}{ TERRAIN } & TERRAIN_SCATTER & Calculates surface reflectivity \\
& TERRAIN_LIT & Finds portions of terrain visible to radar \\
& PN_ORTHOTOMIC & Finds trajectory of image point \\
MICROPHYSICS & PATH_INTEGRAL & Evaluates optical paths between wavefronts \\
and REFRACTION & WS_IMAGE_RAY_QUARTIC & Solves for extremal characteristics \\
& SETLAY & Image of point source in curved surface \\
& & Integrates height of sigma layers \\
\hline
\end{tabular}

Substituting the relation for $s_{12}$ from (A1) we have,

$$
\int_{0}^{L}(d s-d x) \approx \int_{0}^{L} \vartheta^{2} d x+L\left(n\left(\vartheta_{*}\right)-\frac{\vartheta_{*}^{2}}{2}\right)
$$

while,

$$
\int_{0}^{L}(d r-d x) \approx \frac{1}{2} \int_{0}^{L} \vartheta^{2} d x
$$

This latter term is purely geometric and can be calculated from the variational solution of the ray path, using a functional representation of the refractive index profile. Equation (A3) represents the excess phase integral with respect to the free space horizontal path, and comprises twice the geometric term in (A4) plus the last term on the right hand side. This latter term can be evaluated at either end of the path for each ray, knowing the refractive index and slope of the ray. The latter may easily be determined (except where the field vanishes) from the local phase gradient at the ray's launch point. This device avoids the need to integrate the optical path by expressing the refractive index in terms of the actual ray position as a function of the ray parameter.

\section{Appendix B}

This appendix provides an overall description of the software application which implements the methodology described in this paper. The achievement of a software system to simulate realistic radar observations requires the production and integration of significant quantities of computer code. In this case they were written mostly in Fortran 90/95 with a number of distinct applications:

Capability of reading and displaying radar image data

Capability to read NWP model fields and to construct interpolated fields of radar refractivity in radar (polar) coordinates

Capability to utilise high resolution digital elevation models (DEM) and interpolate to radar coordinates

Calculation of ray-trajectories and their intersections with terrain features or sea
Modelling of the distribution of the coherent field distribution from the radar antenna via ground reflections to the extreme range of the radar

Modelling of the terrain or sea reflectivity

Combining modelled imagery with radar and terrain feature imagery

Table B1 contains a list and a brief description of the different modules developed covering some of the above mentioned aspects. The user interface was expected to be a critical component in the evaluation of the technique. Qualitatively, it was useful to be able to see the radar image and model prediction either side by side or overlaid. Since terrain interaction is important in anomalous propagation, it is also important to be able to display terrain and coastal features. An important part of the software system development had therefore been to consider how to achieve this, and to implement the necessary programs to allow the various types of display to be combined. It was also acknowledged that it may only become clear after further experience had been gained in using the package as to what works best in this respect. The software design has taken these factors into account by constructing the front end using the Tcl/tk scripting language (Tool Command Language/Tool Kit extension) which allows rapid reconfiguration of the Graphical User Interface (GUI) and implementing the graphical elements as encapsulated PostScript files. The main graphics application then wrote an enveloping PostScript file that allowed layers to be reshuffled without recomputation. The basic Tcl/tk GUI was written, and its functions were completed by the inclusion of scripts that run the various Fortran executables for the simulation itself, and the graphical displays.

Acknowledgements. This work was partially supported by CARPE DIEM, a research project funded by the European Commission under the 5th FP (Contract $N^{\circ}$ EVG1-CT-2001-0045).

Edited by: P. P. Alberoni

Reviewed by: two referees

\section{References}

Akima, H.: A Method of Univariate Interpolation that Has the Accuracy of a Third-degree Polynomial. ACM Transactions on 
Mathematical Software, 17(3), 341-366, 1991.

Alberoni, P. P., Anderson, T., Mezzasalma, P., Michelson, D. B., and Nanni, S.: Use of the vertical reflectivity profile for identification of anomalous propagation, Meteorol. Appl., 8, 257-266, 2001.

Alberoni, P. P., Ducrocq V., Gregoric G., Haase G., Holleman I., Lindskog M., Macpherson B., Nuret, M., and Rossa A.: Quality and Assimilation of Radar Data for NWP - A Review-, COST 717 document ISBN 92-894-4842-3, 38 pp, 2003.

Bebbington, D.: DARTH EU Project Final Report, Part II. Anomalous Propagation Modelling, Essex University, UK. 18 pp., 1998. [Available upon request from Essex University, Dep. Electronic Systems Engineering, Colchester, CO4 3SQ, UK].

Bech, J.: Observational analysis and numerical modelling of atmospheric propagation conditions of weather radar echoes. $\mathrm{PhD}$ Thesis. University of Barcelona, 2003.

Bech, J., Sairouni, A., Codina, B., Lorente, J., and Bebbington, D.: Weather radar anaprop conditions at a Mediterranean coastal site, Phys. Chem. Earth (B), 25, 829-832, 2000.

Bech, J., Codina, B., Lorente, J., and Bebbington, D.: Monthly and daily variations of radar anomalous propagation conditions: How "normal" is normal propagation? 2nd European Meteorological Radar Conference, Delft, Netherlands, 35-39, 2002.

Bech, J., Codina, B., Lorente, J., and Bebbington, D.: The sensitivity of single polarization weather radar beam blockage correction to variability in the vertical refractivity gradient, J. Atmos. Oceanic Technol., 20, 845-855, 2003.

Bech, J., Codina, B., and Lorente, J.: Forecasting weather radar propagation conditions, Meteorol. Atmos. Phys., doi:10.1007/s00703-006-0211-x, published on-lin 24e October, 2006.

Codina, B., Sairouni, A., Bech, J., and Redaño, A.: Operational Aplication of a nested mesoscale numerical model in Catalonia (Meteo'96 Project). INM/WMO International Symposium of Cyclones and Hazardous Weather in the Mediterranean, ISBN 84-7632-329-8, 657-667, 1997a.

Codina, B., Aran, M., Young, S., and Redaño, A.: Prediction of a Mesoscale Convective System over Catalonia (Northeastern Spain) with a nested Numerical Model, Meteorol. Atmos. Phys., 62, 9-22, 1997b.

Collier, C. G. and Hardaker P.: Using radar in hydrometeorology, Ch.4, 115-129, in Weather Radar, Principles and Advanced Applications. P. Meischner, Ed., Springer, 337 pp., 2003.
Collier, C. G.: Applications of weather radar systems. John Wiley \& Sons, 390 pp., 1996.

James, C. N., Brodzik, S. R., Edmon, H., Houze Jr., R. A., and Yuter, S. E.: Radar Data Processing and Visualization over Complex Terrain, Weather Forecasting, 15(3), 327-338, 2000.

Dierckx P., Curve and Surface Fitting with Splines, Oxford Science Publications, 304 pp., 1995.

Doviak, R. J. and Zrnic, D. S.: Doppler radar and weather observations. Academic Press, 562 pp., 1993.

Fornasiero, A., Alberoni, P. P., Amorati, R., Ferraris, L., and Taramasso, A. C.: Effects of propagation conditions on radar beamground interaction: impact on data quality, ADGEO, 2, Mediterranean Storms, 201-208, 2005.

Haddad, B., Adane, A., Sauvageot, H., Sadouki, L., and Naili, R.: Identification and filtering of rainfall and ground clutter echoes using textural features, Int. J. Remote Sensing, 25, 4641-4656, 2004.

Illingworth, A.: Improved precipitation rates and data quality by using polarimetric measurements. In Weather Radar: Principles and Advanced Applications, edited by: Meischner, P., Springer, Chapter 5, 130-166, 2003.

Joss, J. and Waldvogel, A.: Precipitation measurement and hydrology, a review, Radar in Meteorology, edited by: Atlas, D., Am.Meteorol. Soc., Boston, Chapter 29a, 577-606, 1990.

Koch, S. E., Skillman, W. C., Kocin, P. J., Wetzel, P. J., Brill, K. F., Keyser, D. A., and McCumber, M. C.: Synoptic scale forecast skill and systematic errors in the MASS 2.0 model, Mon. Wea. Rev., 113, 1714-1737, 1985.

Krajewski, W. F. and Vignal, B.: Evaluation of anomalous propagation echo detection in WSR-88D Data: a large sample case study, J. Atmos. Oceanic Technol., 18, 807-814, 2001.

Michelson, D. B., Sunhede, D.: Spurious weather radar echo identification and removal using multisource temperature information, Meteorol. Appl., 11, 1-14, 2004.

Pamment, J. A. and Conway, B. J.: Objective Identification of Echoes Due to Anomalous Propagation in Weather Radar Data, J. Atmos. Oceanic Technol., 15(1), 98-113, 1998.

Sánchez-Diezma, R., Sempere-Torres, D., Delrieu, G., and Zawadki, I.: An improved methodology for ground clutter substitution based on a pre-classification of precipitation types. 30th Internat. Conf. on Radar Meteor., Münich, Germany, Am. Meteorol. Soc., 271-273, 2001. 